\title{
Factores de riesgo de dermatitis atópica en pacientes de consulta de alergia en Matanzas 2016-2017
}

\author{
Risk factors of the atopic dermatitis in patients of municipal allergy \\ consultation in Matanzas 2016-2017
}

Orayne Ekatherina Ricardo Alonso. ${ }^{1}$, María Beatriz Rodríguez Sánchez. ${ }^{2}$, Miriela Hernández Fernández. ${ }^{3}$ \& Maritza Alonso González. ${ }^{4}$

DOl: https://doi.org/10.33262/anatomiadigital.v4i1.1484

\begin{abstract}
.
Background: A traverse descriptive study in 75 patients that attended the Municipal consultation of Alergology in Matanzas was carried out with confirmed diagnosis of Atopic Dermatitis with ages between 1 month and 18 years, of both sexes, in the period of April of 2016 to March of 2017 and that they agreed on participating in the

\section{Resumen.}

Introducción: la atención del paciente con dermatitis atópica en Matanzas, no escapa a la complejidad de las influencias que inciden en su prevención, diagnóstico y tratamiento, constituyendo un problema práctico consultas en el área estudiada. Objetivo: Caracterizar los factores de riesgo de la dermatitis atópica en pacientes

1 Hospital Pediátrico Eliseo Noel Caamaño, Matanzas, Cuba, orayne.mtz@infomed.sld.cu(D) https://orcid.org/0000-0003-0973-1750

2 Hospital Faustino Pérez Hernández, Matanzas, Cuba. alergiaprov.mtz@infomed.sld.cu(D) https://orcid.org/0000-0003-2340-4799

3 Hospital Pediátrico Eliseo Noel Caamaño, Matanzas, Cuba, miriela.mtz@infomed.sld.cu ${ }^{(D)}$ https://orcid.org/0000-0001-8704-1267

${ }^{4}$ Universidad de Ciencias Médicas de Matanzas, Facultad de Ciencias Médicas Juan Guiteras Gener. Matanzas, Cuba, malonso.mtz @infomed.sld.cu (D) https://orcid.org/0000-0002-6236-354x
\end{abstract}


investigation. Objective: To characterize the risk factors of the Atopic Dermatitis in these patients. Methods: Theoretical, empiric and statistical methods were used to achieve to the proposed objectives. An interview to the mothers was carried out through a questionnaire that was made to obtain the information of the perinatal period and pediatric age. Results: The factors that prevailed inside those patients were: the genetic one, the precocious ablactation, the allergic foods consumed by the mothers during the nursing, the psychological factor, the exposition to environmental factors and the exposures to weather changes, with the proper interrelation among them. Conclusions: Some differences were evidenced in the order of the priority among the opposing factors of risk in the bibliography and in the studied patients.

Keywords: Atopic Dermatitis, risk factors, aetiopathogenesis. asistidos en la consulta municipal de Matanzas en el período de abril de 2016 a marzo de 2017. Metodología: Se realizó un estudio descriptivo transversal en pacientes que asistieron a la consulta municipal de Alergología en Matanzas con diagnóstico confirmado de dermatitis atópica de edades comprendidas entre un mes y 18 años, de ambos sexos, en el período de estudio $(\mathrm{N}=75)$ y que dieran su conformidad en participar en la investigación. Se emplearon métodos teóricos, empíricos mediante una entrevista a las madres por un cuestionario confeccionado por los autores para obtener información del período perinatal y edad pediátrica, y estadísticos. Resultados: Los factores que predominaron fueron el genético, la ablactación precoz, los alimentos alergizantes consumidos por las madres durante la lactancia, el factor psicógeno, la exposición a factores ambientales y los cambios de tiempo, encontrándose interrelación entre ellos. Conclusiones: Se evidenciaron algunas diferencias en el orden de la prioridad entre los factores de riesgo encontrados en la bibliografía y en los pacientes estudiados.

Palabras claves: Dermatitis atópica, factores de riesgo, Etiopatogénesis.

\section{Introducción.}

La dermatitis atópica (DA) es una enfermedad inflamatoria crónica de la piel, recidivante, de etiología multifactorial, que se caracteriza por lesiones de morfología y distribución específica según la edad, combinada con piel seca y prurito intenso, asociada frecuentemente a sintomatología respiratoria de origen alérgico. (Dammak A, Guillet S, 2016; Barbarot S, 
Aubert H, Bernier C, Stalder F ,2016; PomavillaDuy MC, Torres Gutama LC ,2015; Cárdenas Medina A. ,2016).

Tiene una incidencia en países desarrollados de un $10 \%$ de la población total, pero se ha estimado que en poblaciones pediátricas la frecuencia se encuentra entre el 10 y $20 \%$, mientras que varía entre un 2 y $8 \%$ en adultos. Constituye un problema en las consultas de alergólogos y dermatólogos debido a su curso persistente, recidivante y a las dificultades del tratamiento, junto al asma bronquial y la rinitis alérgica, esta enfermedad de la piel presenta una elevada prevalencia en Cuba y el mundo. (Buestán, CA. ,2017).

Se posee poca información sobre la epidemiología del eccema o DA si se compara con la disponible sobre el asma y la rinitis alérgica; sin embargo, han sido descritas variaciones geográficas en cuanto a su prevalencia, que concuerdan de manera considerable con las variaciones regionales. Esto sugiere una variación de la tendencia atópica subyacente dentro de cada país. (Buestán, CA. ,2017).

Se realizó un estudio por primera vez en Cuba, utilizando la metodología estandarizada International Study of Asthma and Allergy in Childhood (ISAAC) en la población urbana metropolitana de los municipios Playa y Marianao, ubicados en la provincia La Habana. Se estudió una muestra de niños de 6 a 7 años de edad y adolescentes de 13 a 14 años: 4829 escolares (1 803 y 3026 respectivamente). Se determinó la prevalencia de la DA y sus características tanto en niños como en adolescentes, para una aproximación a la magnitud de este problema de salud y facilitar la prevención y control de esta entidad. (Varona Pérez P, Fabré Ortiz D, Águila R, Corona B, Venero Fernández S, Suárez Medina R. ,2012).

En el incremento de la DA en los últimos años, se han referido variados factores de riesgo. Reconocer los factores de riesgo, los factores desencadenantes o que exacerban los brotes, es un paso importante para hacer una evitación personalizada. Estos factores pueden variar con la edad, el ambiente y con cada estilo de vida social y cotidiano. El clima, la polución, la ropa, la presencia de ácaros del polvo en las casas, cosméticos, ciertas comidas, cambios de dieta, estresantes psicológicos (sudor, infecciones), el estrés emocional y cambios bruscos de temperatura son ejemplos de ello. (Armengot-Carbo M, Hernández-Martín Á, Torrelo A. ,2015; Plötz SG, Wiesender M, Todorova A, Ring J,2014).

La diversidad de factores de riesgo reportados en la bibliografía, evidencia la complejidad de las investigaciones con estos fines, además que la interrelación entre ellos, ofrece un problema que preocupa a los alergólogos para la orientación precisa en cada caso, al verse efectos que se superponen, lo cual necesita trabajos de mayor profundización que caractericen el contexto en el que se desenvuelven los pacientes en los territorios atendidos por la especialidad. La atención del paciente con DA en Matanzas, no escapa a la complejidad de las influencias que inciden en su prevención, diagnóstico y tratamiento, constituyendo un problema práctico en las consultas en el área estudiada. 
Este estudio se justifica, en opinión de los autores por la importancia de los factores de riesgo y su interrelación con el desarrollo de esta enfermedad, las dificultades en su manejo y tratamiento, los insuficientes reportes investigativos de la provincia y en particular en el municipio de Matanzas y la frecuencia con la que asisten pacientes afectados a la consulta de Alergología en el territorio por lo que se propone como objetivo caracterizar los factores de riesgo que inciden en la aparición de la dermatitis atópica en pacientes que asisten a la consulta municipal de Alergología en Matanzas en el período de abril de 2016 a marzo de 2017.

\section{Metodologia.}

Se realizó un estudio descriptivo y transversal. El universo estuvo integrado por los 75 pacientes de ambos sexos, con edades entre 1 mes y 18 años que asistieron a la consulta Municipal de Alergología en Matanzas con diagnóstico confirmado de DA, en el período seleccionado y que dieran su conformidad o sus padres o tutores, en participar en la investigación.

Se emplearon métodos teóricos, empíricos y estadísticos. Se confeccionó un cuestionario aplicado a través de una entrevista a la madre del paciente, para recoger la información del período perinatal y de la edad pediátrica en relación a los factores de riesgo se determinaron las siguientes variables: características de la vivienda, hacinamiento, alergia alimentaria, lactancia materna, contaminantes ambientales, alérgenos e irritantes ambientales, antecedentes de alergia familiar y personal, antecedentes de enfermedades infecciosas y factores psicógenos, que se tuvieron en cuenta en la investigación y el surgimiento de la enfermedad. La entrevista se realizó el día de la consulta.

La planilla de recolección de datos se sometió a criterio de especialistas, con el objetivo de tener en cuenta la experiencia de los médicos que atienden esta especialidad, sobre las variables que se eligen para el estudio. Y Previamente se sometió a un pilotaje para perfeccionar su calidad y comprensión para la aplicación del instrumento. Se cumplieron los principios bioéticos para este tipo de investigación.

Para el análisis estadístico de las variables factores de riesgo, se creó una base de datos mediante el software SPSS, versión 20. Adicionalmente se estudió la posible asociación de factores en los casos de DA estudiados en el contexto del municipio elegido.

\section{Resultados.}

Dentro de las características sociodemográficas se obtuvo que el 53,3\% de los pacientes son del sexo femenino, con un predominio de las féminas entre los que comenzaron con los síntomas antes de los dos años, mientras que en el grupo de 2 a 12 años la mayor frecuencia es el masculino. El $60 \%$ de las madres tenían nivel preuniversitario, el 6,7\% secundaria 
básica, ninguna tiene solo nivel primario y un 33,3\% son universitarias, por lo que todas las madres están escolarizadas.

En la tabla 1 Se puede observar que los antecedentes de DA entre los familiares de primera línea de los pacientes del estudio no fueron mayoría, primando como antecedentes principales el asma y la rinitis. No se refieren en el estudio otros tipos de enfermedades alérgicas en los familiares de los pacientes con DA dentro de sus antecedentes. La enfermedad alérgica más frecuente en las madres es el asma y la rinitis, en el padre igual, pero, con una proporción inferior y en el hermano fue más frecuente el asma y la DA.

Tabla 1. Distribución de los pacientes con DA atendiendo a los antecedentes patológicos familiares de alergia. Municipio Matanzas. 2016-2017

\begin{tabular}{|c|c|c|c|c|c|c|c|c|}
\hline \multirow[t]{2}{*}{ Enfermedad } & \multicolumn{2}{|c|}{ Solo madre } & \multicolumn{2}{|c|}{ Solo padre } & \multicolumn{2}{|c|}{$\begin{array}{c}\text { Solo } \\
\text { hermano }\end{array}$} & \multicolumn{2}{|c|}{ Ninguno } \\
\hline & No. & $\%$ & No. & $\%$ & No. & $\%$ & No. & $\%$ \\
\hline DA & 9 & 12,0 & 2 & 2,7 & 4 & 5,3 & 60 & 80 \\
\hline Rinitis & 18 & 24,0 & 16 & 21,3 & 1 & 1,3 & 21 & 28 \\
\hline $\begin{array}{l}\text { Conjuntivitis } \\
\text { alérgica }\end{array}$ & 0 & 0 & 0 & 0 & 1 & 1,3 & 71 & 94,6 \\
\hline Asma & 20 & 26,6 & 9 & 12 & 5 & 6,6 & 31 & 41,3 \\
\hline $\begin{array}{l}\text { Alergia a } \\
\text { algún } \\
\text { alimento }\end{array}$ & 8 & 10,6 & 6 & 8 & 0 & 0 & 61 & 81,3 \\
\hline $\begin{array}{l}\text { Otra } \\
\text { enfermedad } \\
\text { alérgica }\end{array}$ & 0 & 0 & 0 & 0 & 0 & 0 & 74 & 98,6 \\
\hline
\end{tabular}

Fuente: Elaboración propia

En relación con los antecedentes patológicos personales del niño (Tabla 2) se pudo constatar, que la mayor incidencia la ofreció la rinitis con 36 pacientes, el $48 \%$ de los casos y el asma con 29 (38,7\%). No se recogieron datos significativos sobre las infecciones padecidas por los niños como antecedente, a pesar de ser frecuentes las infecciones cutáneas, sobre todo por estafilococos aureus en esta entidad.

Tabla 2. Distribución de pacientes atendiendo a los antecedentes patológicos personales del niño.2016-2017

\begin{tabular}{lcc}
\hline \multicolumn{1}{c}{ Patología } & Frecuencia & Porciento \\
\hline Rinitis & 36 & 48,0 \\
Asma & 29 & 38,7
\end{tabular}




\begin{tabular}{lcc} 
Conjuntivitis & 4 & 5,3 \\
$\begin{array}{l}\text { Otras } \\
\text { alergias }\end{array}$ & 9 & 12,0 \\
$\begin{array}{l}\text { Infecciosas } \\
\text { Factor }\end{array}$ & 11 & 14,7 \\
psicógeno & 75 & 100 \\
\hline
\end{tabular}

Fuente: Elaboración propia

La exposición a factores ambientales e irritantes que potencian el riesgo de padecer la enfermedad o desencadenar una crisis estuvo ausente en el $4 \%$ de los niños del estudio, contrastando con el $65,3 \%$, que estuvieron expuestos a más de cuatro factores ambientales y de uno a dos factores irritantes. En esta tabla se muestran todas las posibilidades de combinación, refiriéndose las cifras extremas. (Tabla 3).

La mayor importancia la tuvo el contacto con muñecos de peluche, juguete frecuente en la edad pediátrica, le siguió en orden la presencia de cortinas en el hogar, el aire acondicionado y las mascotas. A pesar de que en la mayoría de los casos existen pacientes que se relacionan con los factores ambientales estudiados, los mencionados son los de mayor importancia y en muchos casos coinciden más de uno. Los irritantes predominantes fueron en orden: el perfume, el humo del cigarro y el talco.

Tabla 3. Distribución de factores ambientales e irritantes en los pacientes estudiados. 2016-2017

\begin{tabular}{|c|c|c|c|c|}
\hline \multirow[b]{2}{*}{$\begin{array}{c}\text { Factores } \\
\text { Ambientales. }\end{array}$} & \multicolumn{3}{|c|}{ Factores Irritantes } & \multirow{2}{*}{$\begin{array}{l}\text { Total } \\
\text { Total }\end{array}$} \\
\hline & Ninguno & $\begin{array}{c}\text { Exposición } \\
\text { de } 1 \text { a } 2 \\
\text { factores }\end{array}$ & $\begin{array}{c}\text { Exposición de } 3 \text { a } 4 \\
\text { factores }\end{array}$ & \\
\hline \multirow{2}{*}{ Ninguno } & 3 & 8 & 0 & 11 \\
\hline & $4,0 \%$ & $10,7 \%$ & $0,0 \%$ & $14,7 \%$ \\
\hline $\begin{array}{c}\text { Exposición } \\
\text { de } 1 \text { a } 3\end{array}$ & 1 & 8 & 4 & 13 \\
\hline factores & $1,3 \%$ & $10,7 \%$ & $5,3 \%$ & $17,3 \%$ \\
\hline $\begin{array}{c}\text { Exposición } \\
\text { de } 4 \text { a } 5\end{array}$ & 12 & 49 & 14 & 75 \\
\hline Factores & 16,0 & $65,3 \%$ & $18,7 \%$ & 100 \\
\hline
\end{tabular}

Fuente: Elaboración propia

Otro resultado de este estudio permitió comprobar que ningún niño mantuvo lactancia materna exclusiva hasta los 6 meses, observándose que 10 niños dejaron de recibirla antes 
de 1 mes de nacido. Solo a 22 niños $(29,3 \%)$ le ofrecieron la lactancia artificial después de los seis meses, e incluso a 10 niños recién nacidos. En relación con la introducción de fórmula (puré), 58 pacientes $(77,3 \%)$ la recibieron antes de los 6 meses, incluso entre 1 y 3 meses se les introdujo fórmula a 22 niños $(29,3 \%)$. Los factores exacerbantes relacionados con el debut de la enfermedad que se refirieron más frecuentemente, fueron los cambios de tiempo y las infecciones virales.

El nivel de escolaridad de la madre es de importancia a criterio de los autores, pues está relacionado con el mayor entendimiento de las orientaciones médicas, la percepción de los riesgos y el cumplimiento de las medidas preventivas indicadas.

Los antecedentes patológicos personales que más frecuentemente se refirieron fueron la rinitis y el asma, coincidiendo con la bibliografía consultada en que estas dos enfermedades se asocian con gran frecuencia con la DA. (Dammak A, Guillet S, 2016;, Barbarot S, Aubert $\mathrm{H}_{2}$ Bernier C, Stalder F. ,2016; PomavillaDuy MC, Torres Gutama LC. ,2015; ArmengotCarbo M, Hernández-Martín Á, Torrelo A. ,2015; Lyons JJ, Milner JD, Stone KD,2015), Ue-hara, referido por Dei-Cas y Blanco Q,A, señala que los antecedentes de DA en los familiares de primera línea en los niños, están en un alto por ciento, mientras que en el desarrollo de la investigación los resultados fueron diferentes, ya que es baja la proporción de DA en los familiares (20\%) contrastando con los resultados de su estudio. Esa diferencia, a criterio de los autores, puede estar sustentada por la interrelación del factor genético con otros, lo que puede modificar la expresión de la enfermedad por su carácter multifactorial y los cambios de condiciones de cada paciente. Los autores concuerdan con la importancia de los antecedentes de atopia en los familiares, lo que en el estudio que se realiza en Matanzas, se encontró más presente la rinitis y el asma por parte de madre y padre independiente, pero con mayores cifras recogidas en la madre ( $24 \%$ y $26,6 \%$ respectivamente). Esto constituye una característica de la población estudiada en este trabajo. Los autores sugieren que el agregado de los alimentos en forma precoz debería ser controlado en pacientes con historia familiar de atopia.

A pesar de que en los últimos años ha tomado mucho protagonismo el factor infeccioso como describe la literatura (Barbarot S, Aubert $\mathrm{H}_{2}$ Bernier C, Stalder F. ,2016; PomavillaDuy MC, Torres Gutama LC. ,2015) no se comportó de esa manera en esta investigación, lo cual es otra característica de los hallazgos.

En relación con el factor psicógeno, la influencia de la personalidad está reportada en la bibliografía como factor desencadenante o exacerbante de la DA. En el presente estudio, se pudo comprobar que en la totalidad de los niños se refieren características como hiperactividad, ansiedad, trastornos del sueño y agresividad, coincidiendo con lo reportado en otros estudios, Gómez-de la Fuente E. (2015); Sidbury R, Tom WL, Bergman JN, Cooper KD, Silverman RA, Berger TG, et al, 2014). Se pudo constatar que fueron coincidentes en 
un mismo niño varios trastornos, por lo que se destaca entre los factores de riesgo asociados a la DA.

Es criterio de los autores que la atención a este factor es de vital importancia por la frecuencia con la que está presente y por la influencia que tiene con la enfermedad, pero además por lo que representa en el desarrollo de la personalidad de los niños que se ven afectados y puede repercutir en su adaptación social. Se puede atribuir que el predominio de los factores psicológicos en estos niños depende de la misma enfermedad que padecen y se ha descrito como personalidad atópica. La orientación a la familia y a la escuela puede ser una estrategia de la atención primaria que beneficie la salud psíquica y la calidad de vida de los niños.

Los resultados que se encontraron en este trabajo en relación a la exposición a los factores ambientales e irritantes, no coinciden con los referidos en un estudio reciente multicéntrico donde se observó que los desencadenantes más frecuentes eran los perfumes y productos de higiene personal, seguidos por la ropa, los ácaros del polvo y los cambios bruscos de temperatura. 10, (Armengot-Carbo M, Hernández-Martín Á, Torrelo A. ,2015).

La importancia de los factores ambientales como riesgo en el desarrollo de la DA ha sido referido por autores como Campos,L, Castro A.R, por la presencia de irritantes y productos contaminantes (agentes proinflamatorios) en el aire que afectan las vías respiratorias. (Olsen JR, Gallacher J, Finlay AY, Piguet V, Francis NA, 2016) Estos resultados concuerdan con lo que se obtuvieron en el presente trabajo y refuerza la importancia de este factor. Es criterio de los autores que la influencia del humo del tabaco generalmente no se asocia por la población, al desencadenamiento de enfermedades alérgicas por relacionarse poco en la cultura popular, sin embargo, en el presente estudio hubo una proporción similar entre pacientes expuestos y no expuestos al humo del cigarro.

\section{Conclusiones.}

- Se concluye que se encontró similar proporción de la edad de aparición de la enfermedad en ambos sexos. Los factores de riesgo identificados en este estudio fueron el factor genético, la ablactación precoz, los factores ambientales y los psicógenos. El factor genético fue el más relevante, aparece una elevada prevalencia de asma y rinitis sobre todo de la madre sola. Los antecedentes de DA entre los familiares de primera línea no fueron mayoría. En los antecedentes patológicos personales del niño se destacan la rinitis y el asma, y el factor psicógeno que está presente en todos los niños. Le sigue en orden, la ablactación precoz que tuvieron todos los pacientes, no teniendo ninguno lactancia materna exclusiva hasta los 6 meses, introduciéndose combinación de alimentos. Se manifestó en este estudio también la exposición a factores ambientales e irritantes. Estos factores de riesgo se 
vieron relacionados de forma proporcional, ya que mientras más exposición a estos factores más precozmente se presenta la enfermedad.

\section{Referencias bibliográficas.}

Armengot-Carbo, M., Hernández-Martín, A.,Torrelo, A. (2015).Filagrina: papel en la barrera cutánea y en el desarrollo de patología. Actas Dermo-Sifiliográficas, 106(2). http://www.sciencedirect.com/science/article/pii/S1578219014003333

Barbarot, S., Aubert, H., Bernier, C.,Stalder, F. (2016). Dermatitis atópica. EMCDermatología, $50(4)$ http://www.sciencedirect.com/science/article/pii/S1761289616808924

Buestán, C. A. (2017). Prevalencia de DA en niños que acuden a una guardería de la ciudad de Quito. Rev Facultad Ciencias Médicas, 31 (1-2), 37-39, http://revistadigital.uce.edu.ec/index.php/CIENCIAS_MEDICAS/article/view/485

Cárdenas Medina, A. (2016). DA: los 5 puntos clave para su diagnóstico y manejo. Weidinger S, Novak N. Atopic dermatitis, https://sapiensmedicus.org/puntos-clavesen-la-dermatitis-atopica/

Dammak, A.,Guillet, S. (2016). Dermatitis atópica del niño.EMC-Tratado de Medicina, 19(3), http://www.sciencedirect.com/science/article/pii/S1636541015728242

Gómez-de la Fuente, E. (2015). ¿Se puede prevenir la DA? Actas Dermo-Sifiliográficas, 106 (4), http://www.sciencedirect.com/science/article/pii/S0001731015000071

Lyons, J. J., Milner, J. D.,Stone, K. D.(2015). Atopic Dermatitis in Children: Clinical Features, Pathophysiology, and Treatment. Immunol Allergy Clin North, 35(1), 16183.

Olsen, J. R., Gallacher, J., Finlay, A. Y., Piguet, V.,Francis, N. A.(2016). Quality of Life Impact of Childhood Skin Conditions Measured Using the Children's Dermatology Life Quality Index (CDLQI): A Meta-Analysis. PedriaticDermatology, 174(4), 853861, http://onlinelibrary.wiley.com/doi/10.1111/bjd.14361/full

Plötz, S. G., Wiesender, M., Todorova, A.,Ring, J. (2014). What Is New in Atopic Dermatitis/Eczema? ExpertOpinEmergDrugs, 19(4), 441-458, http://www.tandfonline.com/doi/abs/10.1517/14728214.2014.953927

PomavillaDuy, M. C., Torres Gutama, L. C. (2015). Prevalencia de DA y el estudio de sus comorbilidades en pacientes de 3 meses a 16 años de consulta externa de dermatología del Hospital Vicente Corral Moscoso. Cuenca 2014-2015 (tesis de licenciatura). Universidad de Cuenca, Ecuador, http://dspace.ucuenca.edu.ec/handle/123456789/23652 2015

Sathishkumar, D.,Moss, C. (2016). Topical Therapy in Atopic Dermatitis in Children.Indian J. Dermatol, 61(6), https://www.ncbi.nlm.nih.gov/pmc/articles/PMC5122282/

Sidbury, R., Tom, W. L., Bergman, J. N., Cooper, K. D., Silverman, R. A., Berger, T. G., et al. (2014). Guidelines of Care for the Management of Atopic Dermatitis: Section 4. Prevention of Disease Flares and Use of Adjunctive Therapies and Approaches.J Am AcadDermatol, $71(6)$

1218-1233,

http://www.sciencedirect.com/science/article/pii/S0190962214018878

Simpson, E. L., Irvine, A. D., Eichenfield, L. F.,Friedlander, S. F.(2016).Update on Epidemiology, Diagnosis, and Disease Course of Atopic Dermatitis. SeminCutanMedSurg, $35(5)$ S84-88, 
http://www.ingentaconnect.com/contentone/fmc/scms/2016/00000035/A00505s5/art0 0002

Suárez Ramos, L., León Gómez, O., Sánchez Suárez, E. F.,Bassols Viñas, E. F. (2017).Educational Strategy to Lessen Risk Factors in Children with Atopic Dermatitis.RevHumMed, $17(2)$ http://scielo.sld.cu/scielo.php?script=sci_arttext\&pid=S1727$81202017000200003 \& \operatorname{lng}=\mathrm{es}$

Varona Pérez, P., Fabré Ortiz, D., Águila, R., Corona, B., Venero Fernández, S.,Suárez Medina, R. (2012). Prevalencia de síntomas de DA en niños y adolescentes en La Habana (2002-2003). Rev Cubana Med Gen Integr, 28(1), 42-51, http://scielo.sld.cu/scielo.php?script=sci_arttext\&pid=S086421252012000100006\&lng=es 


\section{PARA CITAR EL ARTÍCULO INDEXADO.}

Ricardo Alonso, O. E., Rodríguez Sánchez, M. B., Hernández Fernández, M., \& Alonso González, M. (2021). Factores de riesgo de dermatitis atópica en pacientes de consulta de alergia en Matanzas 2016-2017 . Anatomía Digital, 4(1), 90-100. https://doi.org/10.33262/anatomiadigital.v4i1.1484

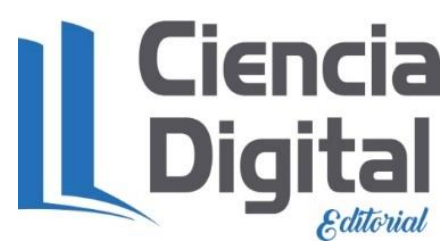

El artículo que se publica es de exclusiva responsabilidad de los autores y no necesariamente reflejan el pensamiento de la Revista Anatomía Digital.

El artículo queda en propiedad de la revista y, por tanto, su publicación parcial y/o total en otro medio tiene que ser autorizado por el director de la Revista Anatomía Digital.
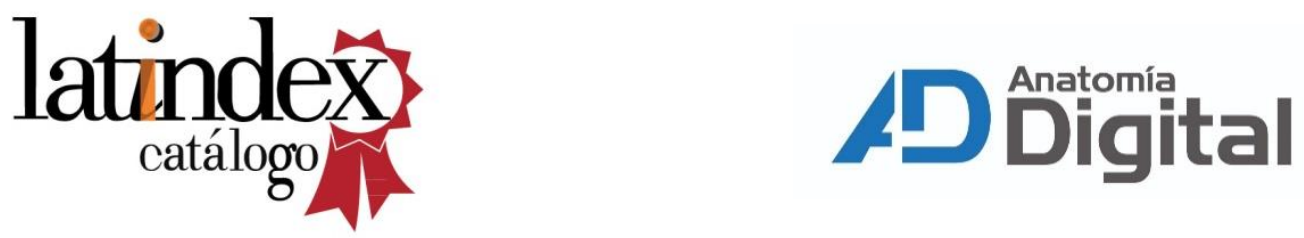